\title{
Linearization of Two Second-Order Ordinary Differential Equations via Fiber Preserving Point Transformations
}

\author{
Sakka Sookmee and Sergey V. Meleshko \\ School of Mathematics, Institute of Science, Suranaree University of Technology, \\ Nakhon Ratchasima 30000, Thailand \\ Correspondence should be addressed to Sergey V. Meleshko, sergey@math.sut.ac.th
}

Received 1 July 2011; Accepted 4 August 2011

Academic Editor: M. Escobedo

Copyright (C) 2011 S. Sookmee and S. V. Meleshko. This is an open access article distributed under the Creative Commons Attribution License, which permits unrestricted use, distribution, and reproduction in any medium, provided the original work is properly cited.

\begin{abstract}
The necessary form of a linearizable system of two second-order ordinary differential equations $y_{1}^{\prime \prime}=f_{1}\left(x, y_{1}, y_{2}, y_{1}^{\prime}, y_{2}^{\prime}\right), y_{2}^{\prime \prime}=f_{2}\left(x, y_{1}, y_{2}, y_{1}^{\prime}, y_{2}^{\prime}\right)$ is obtained. Some other necessary conditions were also found. The main problem studied in the paper is to obtain criteria for a system to be equivalent to a linear system with constant coefficients under fiber preserving transformations. A linear system with constant coefficients is chosen because of its simplicity in finding the general solution. Examples demonstrating the procedure of using the linearization theorems are presented.
\end{abstract}

\section{Introduction}

Almost all physical applications of differential equations are based on nonlinear equations, which in general are very difficult to solve explicitly. Ordinary differential equations play a significant role in the theory of differential equations. In the 19th century, one of the most important problems in analysis was the problem of classification of ordinary differential equations [1-4].

One type of the classification problem is the equivalence problem. Two systems of differential equations are said to be equivalent if there exists an invertible transformation which transforms any solution of one system to a solution of the other system. The linearization problem is a particular case of the equivalence problem, where one of the systems is a linear system. It is one of essential parts in the studies of nonlinear equations. 


\subsection{Linearization Problem}

The analysis of the linearization problem for a single ordinary differential equation was started by Lie [1]. He gave criteria for linearization of a second-order ordinary differential equation by an invertible change of the independent and dependent variables (point transformation). Cartan [4] developed another approach for solving the linearization problem of a second-order ordinary differential equation.

Later the linearization problem was also considered with respect to other types of transformations, for example, contact and generalized Sundman transformations. These transformations have been applied to third-order and fourth-order ordinary differential equations, see [5-16] and references therein. It is worth to note that fiber preserving transformations, where the change of variables only depends on the independent variable, have played a special role: either only such transformations were studied [6], or one needs to study them separately during compatibility analysis [10, 11, 13, 17].

The linearization problem for a system of second-order ordinary differential equations was studied in [18-24]. In [18], necessary and sufficient conditions for a system of $n \geq 2$ second-order ordinary differential equations to be equivalent to the free particle equations were given. Particular class of systems of two $(n=2)$ second-order ordinary differential equations were considered in [20]. In [19], criteria for linearization of a system of two second-order ordinary differential equations are related with the existence of an admitted four-dimensional Lie algebra. Some first-order and second-order relative invariants with respect to point transformations for a system of two ordinary differential equations were obtained in [21]. A new method of linearizing a system of equations is proposed in [22], where a given system of equations is reduced to a single equation to which the Lie theorem on linearization is applied.

\subsection{Canonical Forms of a Linear System of Two Second-Order ODEs}

The general form of a normal linear system of $n$ second-order ordinary differential equations is

$$
\ddot{v}+C \dot{v}+D v+E=0,
$$

where $v=v(t)$ and $E=E(t)$ are vectors, $C=C(t)$ and $D=D(t)$ are $n \times n$ square matrices. It can be shown [19] that there exists a change $u=U v$ such that system (1.1) is reduced to one of the following forms: either

$$
\ddot{u}+K_{1} \dot{u}=0
$$

or

$$
\ddot{u}+K u=0 .
$$

Here $U(t), K=K(t)$, and $K_{1}=K_{1}(t)$ are $n \times n$ square matrices. Thus the problem of linearization via point transformations consists of solving the problem of reducibility of a system of second-order ordinary differential equations to one of these forms. In the present paper, the second canonical form (1.3) is used. 
It is also worth to note that system (1.3) can be further simplified to one of three forms:

$$
K=\left(\begin{array}{ll}
a & 0 \\
0 & b
\end{array}\right), \quad K=\left(\begin{array}{ll}
a & 1 \\
0 & a
\end{array}\right), \quad K=\left(\begin{array}{cc}
a & c \\
-c & a
\end{array}\right),
$$

where $a=a(t), b=b(t)$, and $c=c(t) \neq 0$ are real valued (not complex) functions.

One of the main motivations for studying the linearization problem is the possibility of finding the general solution. Notice that even after finding the linearizing transformation one has to solve a linear system of second-order ordinary differential equations. The simplest case is where $K=0$. More general and also not complicated is the case where the matrix $K$ is constant. For example, for $n=2$, this case leads to solving either a simple linear fourth-order ordinary differential equation with constant coefficients or two simple linear second-order ordinary differential equations. Indeed, for $n=2$, system (1.3) is

$$
\ddot{u}_{1}+k_{1} u_{1}+k_{3} u_{2}=0, \quad \ddot{u}_{2}+k_{4} u_{1}+k_{2} u_{2}=0,
$$

where $k_{i},(i=1,2,3,4)$ are constant. If $k_{3} \neq 0$, then finding $u_{2}$ from the first equation of (1.5) and substituting it into the second equation of (1.5), one obtains a fourth-order ordinary differential equation

$$
u_{1}^{(4)}+\left(k_{1}+k_{2}\right) \ddot{u}_{1}+\left(k_{1} k_{2}-k_{3} k_{4}\right) u_{1}=0 \text {. }
$$

Here,

$$
K=\left(\begin{array}{ll}
k_{1} & k_{3} \\
k_{4} & k_{2}
\end{array}\right)
$$

The general solution of the last equation depends on the roots $\lambda$ of the characteristic equation

$$
\lambda^{4}+\left(k_{1}+k_{2}\right) \lambda^{2}+\left(k_{1} k_{2}-k_{3} k_{4}\right)=0 .
$$

The solution is similar for $k_{4} \neq 0$. On the other hand, if $k_{3}=0$ and $k_{4}=0$, then system (1.1) is decoupled:

$$
\ddot{u}_{1}+k_{1} u_{1}=0, \quad \ddot{u}_{2}+k_{2} u_{2}=0 .
$$

Notice also that if in this case $k_{1}=k_{2}$, then the last system of equations is equivalent to the system of two trivial equations $z^{\prime \prime}=0$.

\subsection{The Problem Solved in the Present Paper}

The present paper deals with a system of two second-order ordinary differential equations

$$
y_{1}^{\prime \prime}=F_{1}\left(x, y_{1}, y_{2}, y_{1}^{\prime}, y_{2}^{\prime}\right), \quad y_{2}^{\prime \prime}=F_{2}\left(x, y_{1}, y_{2}, y_{1}^{\prime}, y_{2}^{\prime}\right)
$$


In the next section, the form of a linearizable system (1.10) is obtained. This form coincides with that obtained in [18]. Some invariants of this form with respect to the general set of point transformations

$$
t=\varphi\left(x, y_{1}, y_{2}\right), \quad u_{1}=\psi_{1}\left(x, y_{1}, y_{2}\right), \quad u_{2}=\psi_{2}\left(x, y_{1}, y_{2}\right)
$$

related with a linearizable systems (1.10) were obtained in [21]. The main result of the paper consists of necessary and sufficient conditions for system (1.10) to be equivalent with respect to a fiber preserving point transformation

$$
t=\varphi(x), \quad u_{1}=\psi_{1}\left(x, y_{1}, y_{2}\right), \quad u_{2}=\psi_{2}\left(x, y_{1}, y_{2}\right)
$$

to system (1.5).

The paper is organized as follows. In Section 2, the necessary form of a linearizable system of two second-order ordinary differential equations is presented. In Section 3, the main results of the paper are given: necessary and sufficient criteria for a system of two second-order ordinary differential equations to be equivalent via fiber preserving point transformations (1.12) to a system of the form (1.5). During the study presented in Section 3, we also obtained some necessary conditions for linearizability for the general case of point transformations (1.11) and for the general case of the matrix $K(t)$. These conditions are shown in Section 4. Examples demonstrating the procedure of using the linearization theorems are presented in Section 5.

\section{Necessary Form of a Linearizable System (1.10)}

For obtaining necessary conditions for system (1.10) to be linearizable via point transformations (1.11), one assumes that system (1.10) is obtained from the linear system of differential equations (1.3) by an invertible transformation (1.11). The derivatives are changed by the formulae

$$
\begin{array}{ll}
u_{1}^{\prime}=g_{1}\left(x, y_{1}, y_{2}, y_{1}^{\prime}, y_{2}^{\prime}\right)=\frac{D_{x} \psi_{1}}{D_{x} \varphi}, & u_{1}^{\prime \prime}=\frac{D_{x} g_{1}}{D_{x} \varphi} \\
u_{2}^{\prime}=g_{2}\left(x, y_{1}, y_{2}, y_{1}^{\prime}, y_{2}^{\prime}\right)=\frac{D_{x} \psi_{2}}{D_{x} \varphi}, & u_{2}^{\prime \prime}=\frac{D_{x} g_{2}}{D_{x} \varphi}
\end{array}
$$

where

$$
D_{x}=\frac{\partial}{\partial x}+y_{1}^{\prime} \frac{\partial}{\partial y_{1}}+y_{2}^{\prime} \frac{\partial}{\partial y_{2}}+y_{1}^{\prime \prime} \frac{\partial}{\partial y_{1}^{\prime}}+y_{2}^{\prime \prime} \frac{\partial}{\partial y_{2}^{\prime}}
$$

Replacing $u_{1}^{\prime}, u_{1}^{\prime \prime}, u_{2}^{\prime}$, and $u_{2}^{\prime \prime}$ in system (1.3), it becomes

$$
\begin{aligned}
& y_{1}^{\prime \prime}=y_{1}^{\prime}\left(a_{11} y_{1}^{\prime 2}+a_{12} y_{1}^{\prime} y_{2}^{\prime}+a_{13} y_{2}^{\prime 2}\right)+a_{14} y_{1}^{\prime 2}+a_{15} y_{1}^{\prime} y_{2}^{\prime}+a_{16} y_{2}^{\prime 2}+a_{17} y_{1}^{\prime}+a_{18} y_{2}^{\prime}+a_{19}, \\
& y_{2}^{\prime \prime}=y_{2}^{\prime}\left(a_{11} y_{1}^{\prime 2}+a_{12} y_{1}^{\prime} y_{2}^{\prime}+a_{13} y_{2}^{\prime 2}\right)+a_{24} y_{1}^{\prime 2}+a_{25} y_{1}^{\prime} y_{2}^{\prime}+a_{26} y_{2}^{\prime 2}+a_{27} y_{1}^{\prime}+a_{28} y_{2}^{\prime}+a_{29},
\end{aligned}
$$


where the coefficients $a_{i j}$ are expressed through the functions $\varphi, \psi_{1}, \psi_{2}$, their partial derivatives, and the entries of the matrix $K=\left(k_{i j}(t)\right)$ as follows:

$$
\begin{aligned}
& a_{11}=\frac{\left(h_{1} \psi_{1} y_{1} y_{1}+h_{2} \psi_{2 y_{1} y_{1}}-v \varphi_{y_{1} y_{1}}+f_{1} \varphi_{y_{1}}{ }^{3}-f_{2} \varphi_{y_{1}}^{2} \varphi_{y_{2}}\right)}{\Delta},
\end{aligned}
$$

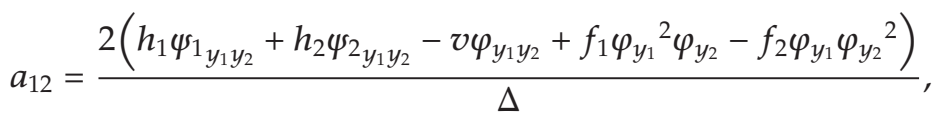

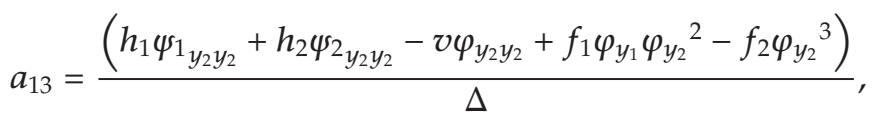

$$
\begin{aligned}
& a_{14}=\frac{\left(2 h_{1} \psi_{1_{x y_{1}}}+2 h_{2} \psi_{2_{x y_{1}}}+h_{3} \psi_{1_{y_{1} y_{1}}}+h_{4} \psi_{2_{y_{1} y_{1}}}+h_{5} \varphi_{y_{1} y_{1}}-2 v \varphi_{x y_{1}}+3 f_{1} \varphi_{x} \varphi_{y_{1}}{ }^{2}-2 f_{2} \varphi_{x} \varphi_{y_{1}} \varphi_{y_{2}}-\mathfrak{J}\right)}{\Delta}, \\
& a_{15}=\frac{2\left(h_{1} \psi_{1_{x y_{2}}}+h_{2} \psi_{2_{x y_{2}}}+h_{3} \psi_{1_{y_{1} y_{2}}}+h_{4} \psi_{2_{y_{1} y_{2}}}+h_{5} \varphi_{y_{1} y_{2}}-2 v \varphi_{x y_{2}}+2 f_{1} \varphi_{x} \varphi_{y_{1}} \varphi_{y_{2}}-f_{2} \varphi_{x} \varphi_{y_{2}}{ }^{2}-\mathfrak{R}\right)}{\Delta}, \\
& a_{16}=\frac{\left(h_{3} \psi_{1} y_{2} y_{2}+h_{4} \psi_{2_{2} y_{2}}+h_{5} \varphi_{y_{2} y_{2}}+f_{1} \varphi_{x} \varphi_{y_{2}}{ }^{2}-f_{3} \varphi_{y_{2}}{ }^{3}\right)}{\Delta}, \\
& a_{17}=\frac{\left(h_{1} \psi_{1 x x}+h_{2} \psi_{2 x x}+2 h_{3} \psi_{1} y_{1}+2 h_{4} \psi_{2 x y_{1}}+2 h_{5} \varphi_{x y_{1}}-v \varphi_{x x}+3 f_{1} \varphi_{x}^{2} \varphi_{y_{1}}-f_{2} \varphi_{x}^{2} \varphi_{y_{2}}-2 f_{3} \varphi_{x} \varphi_{y_{1}}\right)}{\Delta},
\end{aligned}
$$

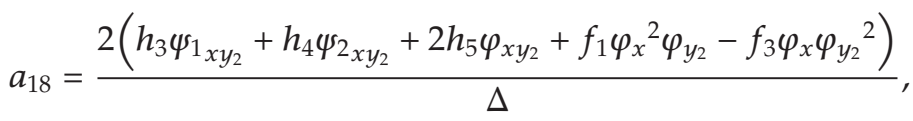

$$
\begin{aligned}
& a_{19}=\frac{\left(h_{3} \psi_{1 x x}+h_{4} \psi_{2 x x}+h_{5} \varphi_{x x}+f_{1} \varphi_{x}^{3}-f_{3} \varphi_{x}^{2} \varphi_{y_{2}}\right)}{\Delta},
\end{aligned}
$$

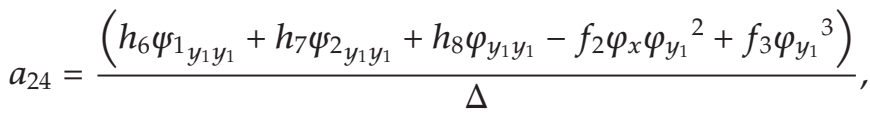

$$
\begin{aligned}
& a_{25}=\frac{2\left(h_{1} \psi_{1_{x y_{1}}}+h_{2} \psi_{2} y_{y_{1}}-v \varphi_{x y_{1}}+h_{6} \psi_{1_{y_{1} y_{2}}}+h_{7} \psi_{2} y_{1} y_{2}+f_{1} \varphi_{x} \varphi_{x} \varphi_{y_{1}}{ }^{2}-2 f_{2} \varphi_{x} \varphi_{y_{1}} \varphi_{y_{2}}-f_{3} \varphi_{y_{1}}{ }^{2} \varphi_{y_{2}}\right)}{\Delta}, \\
& a_{26}=\frac{\left(2 h_{1} \psi_{1_{x y_{2}}}+2 h_{2} \psi_{2 x y_{2}}-2 v \varphi_{x y_{2}}+h_{6} \psi_{1_{y_{2} y_{2}}}+h_{7} \psi_{y_{2} y_{2}}+h_{8} \varphi_{y_{2} y_{2}}+2 f_{1} \varphi_{x} \varphi_{y_{1}} \varphi_{y_{2}}-3 f_{2} \varphi_{x} \varphi_{y_{2}}{ }^{2}-\Re\right)}{\Delta}, \\
& a_{27}=\frac{2\left(h_{6} \psi_{1} y_{y_{1}}+h_{7} \psi_{2} x_{x y_{1}}+h_{8} \varphi_{x y_{1}}-f_{2} \varphi_{x}^{2} \varphi_{y_{1}}+f_{3} \varphi_{x} \varphi_{y_{1}}^{2}\right)}{\Delta}, \\
& a_{28}=\frac{\left(h_{1} \psi_{1_{x x}}+h_{2} \psi_{2 x x}-v \varphi_{x x}+2 h_{6} \psi_{1} y_{y_{2}}+2 h_{7} \psi_{2_{x y_{2}}}+2 h_{8} \varphi_{x y_{2}}+f_{1} \varphi_{x}^{2} \varphi_{y_{1}}-3 f_{2} \varphi_{x}^{2} \varphi_{y_{2}}+\mathfrak{U}\right)}{\Delta}, \\
& a_{29}=\frac{\left(h_{6} \psi_{1_{x x}}+h_{7} \psi_{2_{x x}}+h_{8} \varphi_{x x}-f_{2} \varphi_{x}^{3}+f_{3} \varphi_{x}^{2} \varphi_{y_{1}}\right)}{\Delta},
\end{aligned}
$$


where $\mathfrak{J}$ denotes $f_{3} \varphi_{y_{1}}{ }^{2} \varphi_{y_{2}}, \mathfrak{R}$ denotes $f_{3} \varphi_{y_{1}} \varphi_{y_{2}}{ }^{2}, \mathfrak{U}$ denotes $2 f_{3} \varphi_{x} \varphi_{y_{1}} \varphi_{y_{2}}$, and $\Delta \neq 0$ is the Jacobian of the change of variables (1.11)

$$
\begin{aligned}
& \Delta=\left(\varphi_{x} \psi_{1_{1}} \psi_{2_{y_{2}}}-\varphi_{x} \psi_{1_{y_{2}}} \psi_{2_{y_{1}}}-\varphi_{y_{1}} \psi_{1_{x}} \psi_{2_{y_{2}}}+\varphi_{y_{1}} \psi_{1_{y_{2}}} \psi_{2_{x}}+\varphi_{y_{2}} \psi_{1_{x}} \psi_{2_{y_{1}}}-\varphi_{y_{2}} \psi_{1_{1}} \psi_{2_{x}}\right), \\
& f_{1}=\psi_{1} y_{2}\left(k_{22} \psi_{2}+k_{21} \psi_{1}\right)-\psi_{2} y_{2}\left(k_{11} \psi_{1}+k_{12} \psi_{2}\right), \\
& f_{2}=\psi_{1_{1}}\left(k_{22} \psi_{2}+k_{21} \psi_{1}\right)-\psi_{2 y_{1}}\left(k_{11} \psi_{1}+k_{12} \psi_{2}\right), \\
& f_{3}=\psi_{1}\left(k_{22} \psi_{2}+k_{21} \psi_{1}\right)-\psi_{2 x}\left(k_{11} \psi_{1}+k_{12} \psi_{2}\right), \\
& v=\psi_{1_{2}} \psi_{2} y_{1}-\psi_{1_{1}} \psi_{2 y_{2}}, \quad h_{1}=\varphi_{y_{2}} \psi_{2 y_{1}}-\varphi_{y_{1}} \psi_{2_{y_{2}}} \quad h_{2}=\varphi_{y_{1}} \psi_{1_{y_{2}}}-\varphi_{y_{2}} \psi_{1_{y_{1}}} \\
& h_{3}=\varphi_{y_{2}} \psi_{2 x}-\varphi_{x} \psi_{2_{y_{2}}}, \quad h_{4}=\varphi_{x} \psi_{1_{y_{2}}}-\varphi_{y_{2}} \psi_{1_{x^{\prime}}} \quad h_{5}=\psi_{1_{x}} \psi_{2_{y_{2}}}-\psi_{1_{y_{2}}} \psi_{2_{x^{\prime}}} \\
& h_{6}=\varphi_{x} \psi_{2 y_{1}}-\varphi_{y_{1}} \psi_{2 x_{x}}, \quad h_{7}=\varphi_{y_{1}} \psi_{1_{x}}-\varphi_{x} \psi_{1_{y_{1}}}, \quad h_{8}=\psi_{1_{1}} \psi_{2_{x}}-\psi_{1_{x}} \psi_{2 y_{1}} .
\end{aligned}
$$

Equation (2.3) presents the necessary form of a system of two second-order ordinary differential equations which can be mapped into a linear equation (1.3) via point transformations.

\section{Sufficient Conditions for Equivalency to (1.5) via Fiber Preserving Transformations}

For obtaining sufficient conditions of linearizability of system (2.3), one has to solve the compatibility problem of the system of (2.4), considering it as an overdetermined system of partial differential equations for the functions $\psi_{1}, \psi_{2}$, and $\varphi$ with given coefficients $a_{i j}$ of system (2.3). The compatibility analysis depends on the value of $\psi_{1_{y_{1}}}$.

The next part of the present paper deals with a fiber preserving set of point transformations (1.12):

$$
t=\varphi(x), \quad u_{1}=\psi_{1}\left(x, y_{1}, y_{2}\right), \quad u_{2}=\psi_{2}\left(x, y_{1}, y_{2}\right),
$$

and constant matrix

$$
K=\left(\begin{array}{ll}
k_{1} & k_{3} \\
k_{4} & k_{2}
\end{array}\right)
$$

3.1. Case $\psi_{1_{y_{1}}} \neq 0$

Substitution of $\varphi_{y_{1}}=0$ and $\varphi_{y_{2}}=0$ into (2.4) gives

$$
a_{11}=0, \quad a_{12}=0, \quad a_{13}=0,
$$




$$
\begin{aligned}
& v_{x}=\frac{\left(2 \varphi_{x x} v-\varphi_{x} v\left(a_{17}+a_{28}\right)\right)}{\left(2 \varphi_{x}\right)} \\
& v_{y_{1}}=-\frac{v\left(2 a_{14}+a_{25}\right)}{2}, \\
& v_{y_{2}}=-\frac{v\left(a_{15}+2 a_{26}\right)}{2}, \\
& \psi_{1_{x x}}=\frac{\left(\varphi_{x x} \psi_{1_{x}}-\varphi_{x}^{3}\left(k_{1} \psi_{1}+k_{3} \psi_{2}\right)-\varphi_{x} \psi_{1_{y_{1}}} a_{19}-\varphi_{x} \psi_{1_{y_{2}}} a_{29}\right)}{\varphi_{x}}, \\
& \psi_{1_{x y_{1}}}=\frac{\left(\varphi_{x x} \psi_{1_{y_{1}}}-\varphi_{x} \psi_{1_{y_{1}}} a_{17}-\varphi_{x} \psi_{1_{y_{2}}} a_{27}\right)}{\left(2 \varphi_{x}\right)} \\
& \psi_{1_{x y_{2}}}=\frac{\left(\varphi_{x x} \psi_{1_{y_{2}}}-\varphi_{x} \psi_{1_{y_{1}}} a_{18}-\varphi_{x} \psi_{1_{y_{2}}} a_{28}\right)}{\left(2 \varphi_{x}\right)} \\
& \psi_{1_{y_{1} y_{1}}}=-\left(\psi_{1_{y_{1}}} a_{14}+\psi_{1_{y_{2}}} a_{24}\right), \\
& \psi_{1_{1} y_{2}}=-\frac{\left(\psi_{1_{y_{1}}} a_{15}+\psi_{1_{y_{2}}} a_{25}\right)}{2}, \\
& \psi_{1_{y_{2} y_{2}}}=-\left(\psi_{1_{y_{1}}} a_{16}+\psi_{1_{y_{2}}} a_{26}\right), \\
& \psi_{2 y_{2}}=\frac{\left(\psi_{1_{2}} \psi_{2_{y_{1}}}-v\right)}{\psi_{1_{y_{1}}}} \\
& \psi_{2 y_{1} y_{1}}=\frac{\left(a_{24} v-\psi_{1_{1}} \psi_{2} a_{1} a_{14}-\psi_{1_{y_{2}}} \psi_{2_{y_{1}}} a_{24}\right)}{\psi_{1} y_{1}}, \\
& \psi_{2 x y_{1}}=\frac{\left(\varphi_{x x} \psi_{1_{1}} \psi_{2_{y_{1}}}-\varphi_{x} \psi_{1_{y_{1}}} \psi_{2_{y_{1}}} a_{17}-\varphi_{x} \psi_{1_{y_{2}}} \psi_{2 y_{1}} a_{27}+\varphi_{x} a_{27} v\right)}{\left(2 \varphi_{x} \psi_{1_{y_{1}}}\right)}, \\
& \psi_{2 x x}=\frac{\left(\varphi_{x x} \psi_{1_{y_{1}}} \psi_{2 x}-\varphi_{x}^{3} \psi_{1_{y_{1}}}\left(k_{2} \psi_{2}+k_{4} \psi_{1}\right)-\varphi_{x} \psi_{1_{y_{1}}} \psi_{2_{1}} a_{19}-\varphi_{x} \psi_{1_{y_{2}}} \psi_{2} a_{1} a_{29}+\varphi_{x} a_{29} v\right)}{\left(\varphi_{x} \psi_{1_{y_{1}}}\right)},
\end{aligned}
$$

where $\Delta=-\varphi_{x} v \neq 0$. Comparing the mixed derivatives $\left(v_{x}\right)_{y_{1}}=\left(v_{y_{1}}\right)_{x},\left(v_{x}\right)_{y_{2}}=\left(v_{y_{2}}\right)_{x}$ and $\left(v_{y_{2}}\right)_{y_{1}}=\left(v_{y_{1}}\right)_{y_{2}}$, one obtains

$$
\begin{gathered}
2 a_{14 x}-a_{17 y_{1}}+a_{25 x}-a_{28 y_{1}}=0, \quad a_{15 x}-a_{17 y_{2}}+2 a_{26 x}-a_{28 y_{2}}=0, \\
a_{15 y_{1}}-2 a_{14 y_{2}}-a_{25 y_{2}}+2 a_{26 y_{1}}=0 .
\end{gathered}
$$


Considering the conditions $\left(\psi_{i_{x x}}\right)_{y_{j}}=\left(\psi_{i_{x y_{j}}}\right)_{x}(i, j=1,2)$, one has

$$
\begin{aligned}
& \varphi_{x x x}=\frac{\left(3 \varphi_{x x}{ }^{2} \psi_{1_{1}}-4 \varphi_{x}^{4} \psi_{1_{1}} k_{1}-4 \varphi_{x}^{4} \psi_{2_{y_{1}}} k_{3}+\varphi_{x}^{2} \psi_{1_{y_{1}}}\left(\lambda_{20}-\lambda_{16}\right)+\varphi_{x}^{2} \psi_{1_{y_{2}}} \lambda_{12}\right)}{\left(2 \varphi_{x} \psi_{1}\right)}, \\
& k_{4}=\frac{\left(4 \varphi_{x}^{2} \psi_{1_{y_{1}}} \psi_{2_{y_{1}}}\left(k_{1}-k_{2}\right)+4 \varphi_{x}{ }^{2} \psi_{2 y_{1}}{ }^{2} k_{3}-v \lambda_{12}\right)}{\left(4 \varphi_{x}{ }^{2} \psi_{1_{y_{1}}}{ }^{2}\right)}, \\
& k_{3}=\frac{\left(\psi_{1_{y_{2}}}{ }^{2} \lambda_{12}-\psi_{1_{y_{1}}}{ }^{2} \lambda_{15}-\psi_{1_{y_{1}}} \psi_{\left.1_{y_{2}} \lambda_{16}\right)}\right.}{\left(4 \varphi_{x}{ }^{2} v\right)} \\
& k_{2}=\frac{\left(4 \varphi_{x}^{2} \psi_{1_{1}} k_{1} v-2 \psi_{1_{y_{1}}}^{2} \psi_{2} \lambda_{y_{1}} \lambda_{15}-2 \psi_{1_{y_{1}}} \psi_{1_{y_{2}}} \psi_{2_{y_{1}}} \lambda_{16}+\psi_{1_{1}} v \lambda_{16}+2 \psi_{1_{y_{2}}}^{2} \psi_{2} \lambda_{y_{1}} \lambda_{12}-2 \psi_{1_{y_{2}}} v \lambda_{12}\right)}{\left(4 \varphi_{x}{ }^{2} \psi_{1_{1}} v\right)} .
\end{aligned}
$$

Here, the functions $\lambda_{n}\left(x, y_{1}, y_{2}\right)$ are defined through $a_{i j}\left(x, y_{1}, y_{2}\right)$ and their derivatives (presented in the appendix).

Equating the mixed derivatives $\left(\psi_{i_{x y_{1}}}\right)_{y_{2}}=\left(\psi_{i_{x y_{2}}}\right)_{y_{1}},\left(\psi_{i_{x y_{1}}}\right)_{y_{1}}=\left(\psi_{i_{y_{1} y_{1}}}\right)_{x},\left(\psi_{1_{x y_{2}}}\right)_{y_{i}}=$ $\left(\psi_{1} y_{i} y_{2}\right)_{x},\left(\psi_{1} y_{1} y_{i}\right)_{y_{2}}=\left(\psi_{1} y_{i} y_{2}\right)_{y_{1}},\left(\psi_{2} y_{1} y_{1}\right)_{y_{2}}=\left(\psi_{2 y_{2}}\right)_{y_{1} y_{1}}$ and using the conditions $\left(k_{i+1}\right)_{y_{2}}=0$, $(i=1,2)$, one obtains

$$
\lambda_{n}=0 \quad(n=1,2, \ldots, 11)
$$

Note that $\left(\psi_{1} x_{x y_{2}}\right)_{y_{1}}-\left(\psi_{1} y_{1 y_{2}}\right)_{x}=0$ is satisfied. Differentiating (3.16) and (3.17) with respect to $y_{1}$ and $y_{2}$, one has

$$
2 \lambda_{15 y_{1}}-2 a_{14} \lambda_{15}-a_{15} \lambda_{16}+a_{25} \lambda_{15}=0, \quad \lambda_{27+j}=0 \quad(j=0,1,2) .
$$

Equations $\left(k_{i}\right)_{x}=0(i=2,3,4)$, become

$$
\begin{gathered}
4 \varphi_{x x} \lambda_{12}+\varphi_{x} \lambda_{14}=0 \\
2 \varphi_{x x} \lambda_{16}-\varphi_{x}\left(\lambda_{16 x}+a_{18} \lambda_{12}-a_{27} \lambda_{15}\right)=0 \\
4 \varphi_{x x} \lambda_{15}+\varphi_{x}\left(a_{17} \lambda_{15}-2 \lambda_{15 x}+a_{18} \lambda_{16}-a_{28} \lambda_{15}\right)=0 .
\end{gathered}
$$

Further analysis of the compatibility depends on the values of the coefficients $\lambda_{12}, \lambda_{16}$ and $\lambda_{15}$ of the last three equations (3.21)-(3.23). 


\subsubsection{Case $\lambda_{12} \neq 0$}

Substituting $\varphi_{x x}$, found from (3.21), into (3.23) and (3.22), one obtains

$$
\begin{gathered}
a_{17} \lambda_{12} \lambda_{15}-2 \lambda_{15 x} \lambda_{12}+a_{18} \lambda_{12} \lambda_{16}-a_{28} \lambda_{12} \lambda_{15}-\lambda_{14} \lambda_{15}=0, \\
2 \lambda_{16 x} \lambda_{12}+2 a_{18} \lambda_{12}^{2}-2 a_{27} \lambda_{12} \lambda_{15}+\lambda_{14} \lambda_{16}=0 .
\end{gathered}
$$

Differentiating (3.21) with respect to $y_{1}$ and $y_{2}$, one has

$$
\lambda_{12 y_{1}} \lambda_{14}-\lambda_{14 y_{1}} \lambda_{12}=0, \quad \lambda_{12 y_{2}} \lambda_{14}-\lambda_{14 y_{2}} \lambda_{12}=0
$$

Equation (3.16) becomes

$$
\begin{aligned}
k_{1}=( & 16 \psi_{1_{1}}{ }^{2} \psi_{2_{y_{1}}} \lambda_{12}{ }^{2} \lambda_{15}+16 \psi_{1_{y_{1}}} \psi_{1_{y_{2}}} \psi_{2_{y_{1}}} \lambda_{12}{ }^{2} \lambda_{16} \\
& +16 \psi_{1_{y_{1}}} \lambda_{12}{ }^{2} v\left(\lambda_{20}-\lambda_{16}\right)+4 \psi_{1_{1}} \lambda_{12} v\left(2 \lambda_{14 x}+a_{17} \lambda_{14}-a_{28} \lambda_{14}\right) \\
& \left.+\psi_{1_{y_{1}}} \lambda_{14} v\left(4 a_{27} \lambda_{16}+5 \lambda_{14}\right)-16 \psi_{1_{y_{2}}}{ }^{2} \psi_{2}{ }_{y_{1}} \lambda_{12}{ }^{3}+16 \psi_{1_{y_{2}}} \lambda_{12}{ }^{3} v\right) /\left(64 \varphi_{x}{ }^{2} \psi_{1_{y_{1}}} \lambda_{12}{ }^{2} v\right) .
\end{aligned}
$$

Differentiating (3.26) with respect to $x$, one gets the condition

$$
32 \lambda_{12}{ }^{3} \lambda_{17}+8 \lambda_{12}{ }^{2} \lambda_{18}+2 \lambda_{12} \lambda_{19}+\lambda_{14}\left(8 a_{27}{ }^{2} \lambda_{16}{ }^{2}+18 a_{27} \lambda_{14} \lambda_{16}+15 \lambda_{14}{ }^{2}\right)=0 .
$$

Notice that $\left(k_{1}\right)_{y_{1}}=0$ and $\left(k_{1}\right)_{y_{2}}=0$ are satisfied. Hence, there are no new conditions for the functions $\varphi(x), \psi_{1}\left(x, y_{1}, y_{2}\right)$, and $\psi_{2}\left(x, y_{1}, y_{2}\right)$. In summary, the criteria for linearization are conditions (3.3), (3.15), (3.19), (3.20), (3.24), (3.25), and (3.27).

\subsubsection{Case $\lambda_{12}=0$ and $\lambda_{16} \neq 0$}

Since $\lambda_{12}=0$ and $\varphi_{x} \neq 0,(3.21)$ leads to the condition $\lambda_{14}=0$, and (3.22) becomes

$$
\varphi_{x x}=\frac{\varphi_{x} \lambda_{16 x}}{\left(2 \lambda_{16}\right)}
$$

Substituting $\varphi_{x x}$ into (3.23) and (3.16), one gets

$$
\begin{gathered}
2\left(\lambda_{16 x} \lambda_{15}-\lambda_{15 x} \lambda_{16}\right)+\lambda_{15} \lambda_{16}\left(a_{17}-a_{28}\right)+a_{18} \lambda_{16}{ }^{2}=0 \\
k_{1}=\frac{\left(4 \psi_{1_{1}} \psi_{2 y_{1}} \lambda_{16}{ }^{2} \lambda_{15}+4 \psi_{1}{ }_{y_{2}} \psi_{2 y_{1}} \lambda_{16}{ }^{3}-4 \lambda_{16}{ }^{3} v+4 \lambda_{16}{ }^{2} \lambda_{23} v-4 \lambda_{16} \lambda_{16 x x} v+5 \lambda_{16 x} v\right)}{\left(16 \varphi_{x}{ }^{2} \lambda_{16}{ }^{2} v\right)} .
\end{gathered}
$$


Equation $\left(k_{1}\right)_{x}=0$ leads to the condition

$$
8 \lambda_{16}{ }^{3} \lambda_{21}+4 \lambda_{16}{ }^{2} \lambda_{22}+18 \lambda_{16} \lambda_{16 x x} \lambda_{16 x}-15 \lambda_{16 x}{ }^{3}=0 .
$$

Note that $\left(\varphi_{x x}\right)_{y_{i}}=0$ and $\left(k_{1}\right)_{y_{i}}=0,(i=1,2)$ are satisfied. Hence, there are no other conditions for the functions $\varphi(x), \psi_{1}\left(x, y_{1}, y_{2}\right)$, and $\psi_{2}\left(x, y_{1}, y_{2}\right)$. Summarizing, the linearization criteria in the case $\lambda_{12}=0$ and $\lambda_{16} \neq 0$ are conditions (3.3), (3.15), (3.19), (3.20), (3.29), and (3.31).

\subsubsection{Case $\lambda_{12}=0, \lambda_{16}=0$, and $\lambda_{15} \neq 0$}

Substituting $\varphi_{x x}$, found from (3.23), into (3.16), one has

$$
k_{1}=\frac{\left(16 \psi_{1}{ }_{y_{1}} \psi_{2}{ }_{y_{1}} \lambda_{15}{ }^{3}+\lambda_{26} v\right)}{\left(64 \varphi_{x}{ }^{2} \lambda_{15}{ }^{2} v\right)} .
$$

Differentiating (3.32) with respect to $x$, one gets

$$
\lambda_{15}{ }^{3} \lambda_{24}+2 \lambda_{15}{ }^{2} \lambda_{25}-120 \lambda_{15 x}{ }^{3}+36 \lambda_{15} \lambda_{15 x}\left(4 \lambda_{15 x x}+\lambda_{15 x} a_{17}-\lambda_{15 x} a_{28}\right)=0 .
$$

Note that the equations $\left(\varphi_{x x}\right)_{y_{i}}=0$ and $\left(k_{1}\right)_{y_{i}}=0,(i=1,2)$ are satisfied. Hence, there are no more conditions for the compatibility, and the linearization criteria in the studied case are (3.3), (3.15), (3.19), (3.20) and (3.33).

Remark 3.1. In the case $\lambda_{12}=0, \lambda_{16}=0$ and $\lambda_{15}=0$, one has $k_{2}=k_{1}$ and $k_{3}=k_{4}=0$. This case corresponds to (1.9).

Combining all derived results in the case $\psi_{1_{1}} \neq 0$, the following theorem is proven.

Theorem 3.2. Necessary and sufficient conditions for system (2.3) to be equivalent to a linear system (1.3) with constant matrix $K$ via fiber preserving transformations are as follows.

(I) The conditions are (3.3), (3.15), (3.19), and (3.20), and the additional conditions are as

(I.1) If $\lambda_{12} \neq 0$, then the additional conditions are (3.24), (3.25), and (3.27).

(I.2) If $\lambda_{12}=0$ and $\lambda_{16} \neq 0$, then the additional conditions are (3.29) and (3.31).

(I.3) If $\lambda_{12}=0, \lambda_{16}=0$, and $\lambda_{15} \neq 0$, then the additional condition is (3.33).

(I.4) If $\lambda_{12}=0, \lambda_{16}=0$, and $\lambda_{15}=0$, then there are no additional conditions. 


\subsection{Case $\psi_{1_{y_{1}}}=0$}

If $\psi_{2_{y_{2}}} \neq 0$, then the change $\bar{x}=x, \overline{y_{1}}=y_{2}, \overline{y_{2}}=y_{1}$ leads to the previous case. Hence, without loss of generality one can assume that $\psi_{2} y_{2}=0$. Equations (2.4) become

$$
\begin{aligned}
& a_{11}=0, \quad a_{12}=0, \quad a_{13}=0, \quad a_{15}=0, a_{16}=0, \quad a_{18}=0, a_{24}=0, a_{25}=0, a_{27}=0, \\
& \psi_{2 y_{1} y_{1}}=-\psi_{2 y_{1}} a_{14}, \quad \psi_{2 x y_{1}}=\frac{\left(\varphi_{x x} \psi_{2_{y_{1}}}-\varphi_{x} \psi_{2 y_{1}} a_{17}\right)}{\left(2 \varphi_{x}\right)}, \\
& \psi_{1_{y_{2} y_{2}}}=-\psi_{1_{y_{2}}} a_{26}, \quad \psi_{1_{x y_{2}}}=\frac{\left(\varphi_{x x} \psi_{1_{y_{2}}}-\varphi_{x} \psi_{1_{y_{2}}} a_{28}\right)}{\left(2 \varphi_{x}\right)}, \\
& \psi_{1_{x x}}=\frac{\left(\varphi_{x x} \psi_{1_{x}}-\varphi_{x}^{3}\left(k_{1} \psi_{1}+k_{3} \psi_{2}\right)-\varphi_{x} \psi_{1_{2}} a_{29}\right)}{\varphi_{x}}, \\
& \psi_{2 x x}=\frac{\left(\varphi_{x x} \psi_{2 x}-\varphi_{x}^{3}\left(k_{2} \psi_{2}+k_{4} \psi_{1}\right)-\varphi_{x} \psi_{2 y_{1}} a_{19}\right)}{\varphi_{x}},
\end{aligned}
$$

and $\Delta=-\varphi_{x} \psi_{1_{y_{2}}} \psi_{2 y_{1}} \neq 0$.

Comparing all mixed derivatives of third-order of the functions $\psi_{j}, j=1,2$, one finds

$$
\begin{array}{cc}
a_{28 y_{1}}=0, \quad a_{28 y_{2}}=2 a_{26 x}, \quad a_{26 y_{1}}=0, & a_{17 y_{2}}=0, \quad a_{17 y_{1}}=2 a_{14 x}, \quad a_{14 y_{2}}=0, \\
k_{1}=\frac{\left(3 \varphi_{x x}^{2}-2 \varphi_{x x x} \varphi_{x}+\varphi_{x}^{2} \mu_{1}\right)}{\left(4 \varphi_{x}^{4}\right)}, & k_{2}=\frac{\left(3 \varphi_{x x}^{2}-2 \varphi_{x x x} \varphi_{x}-\varphi_{x}^{2} \mu_{2}\right)}{\left(4 \varphi_{x}^{4}\right)}, \\
k_{3}=\frac{\left(-a_{29} y_{1} \psi_{1 y_{2}}\right)}{\left(\varphi_{x}^{2} \psi_{2 y_{1}}\right)}, & k_{4}=\frac{\left(-a_{19 y_{2}} \psi_{2 y_{1}}\right)}{\left(\varphi_{x}^{2} \psi_{1 y_{2}}\right)},
\end{array}
$$

where the coefficients $\mu_{n}$ are defined through $a_{i j}$ and their derivatives (presented in the appendix).

Equations $\left(k_{i}\right)_{x}=0,\left(k_{i}\right)_{y_{j}}=0(i=1,2,3,4, j=1,2)$, become

$$
\begin{gathered}
\varphi_{x x x x}=\frac{\left(12 \varphi_{x x x} \varphi_{x x} \varphi_{x}-12 \varphi_{x x}^{3}+2 \varphi_{x x} \varphi_{x}^{2} \mu_{2}-\varphi_{x}^{3} \mu_{2_{x}}\right)}{\left(2 \varphi_{x}^{2}\right)}, \\
\left(\varphi_{x x}-\varphi_{x} \mu_{3}\right) a_{29 y_{1}}=0, \quad\left(\varphi_{x x}-\varphi_{x} \mu_{4}\right) a_{19 y_{2}}=0, \quad 2 \varphi_{x x} \mu_{5}-\varphi_{x} \mu_{5 x}=0, \\
a_{19 y_{2}} a_{14}-a_{19 y_{1} y_{2}}=0, \quad a_{29 y_{1}} a_{26}-a_{29 y_{1} y_{2}}=0, \\
a_{29 y_{1} y_{1}}+a_{29 y_{1}} a_{14}=0, \quad a_{19 y_{2} y_{2}}+a_{19 y_{2}} a_{26}=0, \\
a_{26 x x}-a_{26 x} a_{28}+a_{26 y_{2}} a_{29}-a_{29 y_{2} y_{2}}+a_{29 y_{2}} a_{26}=0, \\
a_{14 x x}-a_{14 x} a_{17}+a_{14 y_{1}} a_{19}-a_{19 y_{1} y_{1}}+a_{19 y_{1}} a_{14}=0 .
\end{gathered}
$$


Notice that $\left(\varphi_{x x x x}\right)_{y_{j}}=0(j=1,2)$ are satisfied. Equation (3.39) generates a further analysis of the compatibility depending on the values of $a_{29} y_{1}, a_{19 y_{2}}$, and $\mu_{5}$.

\subsubsection{Case $a_{29} y_{1} \neq 0$}

From the first equation of (3.39), one obtains that

$$
\varphi_{x x}=\varphi_{x} \mu_{3}
$$

Substituting $\varphi_{x x}$ into the second and third equations of (3.39), one obtains

$$
2 \mu_{5} \mu_{3}-\mu_{5 x}=0, \quad a_{19 y_{2}}\left(\mu_{3}-\mu_{4}\right)=0
$$

Substitution of $\varphi_{x x}$ into (3.38) gives

$$
2 \mu_{3 x x}+\mu_{2 x}-6 \mu_{3 x} \mu_{3}-2 \mu_{2} \mu_{3}+2 \mu_{3}^{3}=0
$$

Note that $\left(\varphi_{x x}\right)_{y_{1}}=0$ and $\left(\varphi_{x x}\right)_{y_{2}}=0$ are satisfied. Hence, there are no new conditions. In summary, the linearization criteria are (3.34), (3.36), (3.40), (3.42), and (3.43).

\subsubsection{Case $a_{29} y_{1}=0$ and $a_{19 y_{2}} \neq 0$}

From the second equation of (3.39), one obtains that

$$
\varphi_{x x}=\varphi_{x} \mu_{4}
$$

Substituting $\varphi_{x x}$ into the third equation of (3.39), one obtains

$$
\mu_{5 x}-2 \mu_{5} \mu_{4}=0
$$

Substitution of $\varphi_{x x}$ into (3.38) gives

$$
6 \mu_{4 x} \mu_{4}-\mu_{2 x}-2 \mu_{4 x x}+2 \mu_{2} \mu_{4}-2 \mu_{4}^{3}=0
$$

Note that $\left(\varphi_{x x}\right)_{y_{1}}=0$ and $\left(\varphi_{x x}\right)_{y_{2}}=0$ are satisfied. Hence, there are no other conditions. Thus, the linearization criteria in this case are (3.34), (3.36), (3.40), (3.45), and (3.46).

\subsubsection{Case $a_{29} y_{1}=0, a_{19} y_{2}=0$, and $\mu_{5} \neq 0$}

From the third equation of (3.39), one obtains

$$
\varphi_{x x}=\frac{\left(\varphi_{x} \mu_{5_{x}}\right)}{\left(2 \mu_{5}\right)} .
$$


Substitution of $\varphi_{x x}$ into (3.38) leads to the condition

$$
\mu_{5}{ }^{2}\left(4 \mu_{5 x} \mu_{2}-4 \mu_{5 x x}\right)+18 \mu_{5 x x} \mu_{5 x} \mu_{5}-15 \mu_{5 x}{ }^{3}-4 \mu_{2 x} \mu_{5}{ }^{3}=0 .
$$

Note that $\left(\varphi_{x x}\right)_{y_{1}}=0$ and $\left(\varphi_{x x}\right)_{y_{2}}=0$ are satisfied. Hence, there are no more conditions. In brief, the linearization criteria are conditions (3.34), (3.36), (3.40), and (3.48). Notice also that

$$
k_{2}=k_{1}+\frac{\mu_{5}}{\left(4 \varphi_{x}^{2}\right)}, \quad k_{3}=0, \quad k_{4}=0
$$

Remark 3.3. In the case $a_{29} y_{1}=0, a_{19} y_{2}=0, \mu_{5}=0$, one has $k_{2}=k_{1}$ and $k_{3}=k_{4}=0$. This case corresponds to (1.9). is proven.

Combining all obtained results in the case $\psi_{1_{1}}=0$ and $\psi_{2} y_{2}=0$, the following theorem

Theorem 3.4. Necessary and sufficient conditions for system (2.3) to be equivalent to a linear system (1.3) with constant matrix $K$ by fiber preserving transformations are as follows.

(II) The conditions are (3.34), (3.36), and (3.40), and the additional conditions are as

(II.1) If $a_{29} y_{1} \neq 0$, then the additional conditions are (3.42) and (3.43).

(II.2) If $a_{29} y_{1}=0$ and $a_{19 y_{2}} \neq 0$, then the additional conditions are (3.45) and (3.46).

(II.3) If $a_{29} y_{1}=0, a_{19 y_{2}}=0$, and $\mu_{5} \neq 0$, then the additional condition is (3.48).

(II.4) If $a_{29} y_{1}=0, a_{19 y_{2}}=0$, and $\mu_{5}=0$, then there are no additional conditions.

Remark 3.5. If one assumes that the conditions (II) of Theorem 3.4 are valid, then the conditions (I) of Theorem 3.2 vanish. Moreover, these conditions also imply that $\lambda_{12}=$ $-4 a_{29} y_{1}, \lambda_{15}=-4 a_{19 y_{2}}, \lambda_{16}=-\mu_{5}$, and the following is valid: (a) the conditions (II.1) become a particular case of the conditions (I.1); (b) the conditions (II.3) are a particular case of the conditions (I.2); (c) the conditions (II.2) with $\mu_{5} \neq 0$ and $\mu_{5}=0$ form particular cases of the conditions (I.2) and (I.3), respectively. This allows to propose the conjecture that Theorem 3.2 is valid independently of the values of $\psi_{1 y_{1}}$ and $\psi_{2 y_{2}}$.

Notice that this conjecture is to be expected. For example, for a linearizable single second-order equation via a point transformation, the linearizable criteria combine to only two conditions, whereas during compatibility analysis, one has to study two separable cases [17].

\section{Necessary Conditions of Linearization under Point Transformations}

During the study presented in the previous section, several relations for linearizability for the general case of point transformations (1.11) and for the general case of the matrix $K(t)$ were noted. These relations are the necessary conditions for linearization, and they were obtained as follows. For example, assuming that $\psi_{1} y_{1} \neq 0$, from (2.4), one obtains the derivatives 
$v_{x}, v_{y_{j}}, \varphi_{x x}, \varphi_{x y_{j}}, \varphi_{y_{j} y_{k}}, \psi_{1} x_{x}, \psi_{l_{x y_{j}}} \psi_{l l_{y_{j} y_{k}}}(j, k, l=1,2)$. Comparing the mixed derivatives of the functions $v, \varphi, \psi_{1}$, and $\psi_{2}$, one can find the expressions of the quantities

$$
\omega_{n} \quad(n=1,2, \ldots, 15),
$$

where $\omega_{n}$ are expressed through $a_{i j}$ and their derivatives (shown in the appendix). Excluding the functions $v, \varphi, \psi_{1}$, and $\psi_{2}$ from these expressions, one obtains the conditions

$$
J_{i}=0 \quad(i=1,2, \ldots, 15),
$$

where

$$
\begin{gathered}
J_{1}=\omega_{1} \omega_{11}-2 \omega_{1} \omega_{9}+2 \omega_{10} \omega_{2}-\omega_{3} \omega_{6}, \quad J_{2}=\omega_{1} \omega_{5}+2 \omega_{2} \omega_{6}, \\
J_{3}=6 \omega_{1} \omega_{8}-2 \omega_{1} \omega_{12}+10 \omega_{11} \omega_{2}-20 \omega_{2} \omega_{9}-5 \omega_{3}^{2}, \\
J_{4}=2 \omega_{10} \omega_{2}-\omega_{1} \omega_{9}, \quad J_{5}=10 \omega_{1} \omega_{7}+\omega_{12} \omega_{2}-3 \omega_{2} \omega_{8}, \\
J_{6}=\omega_{1} \omega_{8}+\omega_{11} \omega_{2}-3 \omega_{2} \omega_{9}, \quad J_{7}=4 \omega_{1} \omega_{12}-2 \omega_{1} \omega_{8}+10 \omega_{3}^{2}+5 \omega_{3} \omega_{5}, \\
J_{8}=10 \omega_{1} \omega_{13}+\omega_{12} \omega_{3}-3 \omega_{3} \omega_{8}, \quad J_{9}=\omega_{1} \omega_{15}-\omega_{10} \omega_{3}, \\
J_{10}=\omega_{1} \omega_{14}+\omega_{11} \omega_{3}-3 \omega_{3} \omega_{9}, \quad J_{11}=2 \omega_{12} \omega_{2}-\omega_{2} \omega_{8}+5 \omega_{3} \omega_{4}, \\
J_{12}=\omega_{13} \omega_{2}-\omega_{3} \omega_{7}, \quad J_{13}=2 \omega_{15} \omega_{2}-\omega_{3} \omega_{9}, \\
J_{14}=\omega_{14} \omega_{2}-\omega_{3} \omega_{8}, \quad J_{15}=2 \omega_{1} \omega_{4}-2 \omega_{2} \omega_{3}-\omega_{2} \omega_{5} .
\end{gathered}
$$

After obtaining these relations, one can directly check, by substituting (2.4) into (4.2), that they are satisfied for the general case of point transformations (1.11) and for the general case of the matrix $K(t)$. Notice also that using this substitution into the conditions obtained in $[18,23]$, one obtains that they are not satisfied unless the matrix $K=0$.

Thus, the following theorem can be stated.

Theorem 4.1. The conditions (4.2) are necessary for system (2.3) to be linearizable under point transformations.

\section{Examples}

In this section, examples demonstrating the procedure of using the linearization theorems are presented.

Example 5.1. Let us consider a system of two second-order quadratically semilinear ordinary differential equations

$$
\begin{aligned}
& y_{1}^{\prime \prime}=a\left(y_{1}, y_{2}\right) y_{1}^{\prime 2}+2 b\left(y_{1}, y_{2}\right) y_{1}^{\prime} y_{2}^{\prime}+c\left(y_{1}, y_{2}\right) y_{2}^{\prime 2}, \\
& y_{2}^{\prime \prime}=d\left(y_{1}, y_{2}\right) y_{1}^{\prime 2}+2 e\left(y_{1}, y_{2}\right) y_{1}^{\prime} y_{2}^{\prime}+f\left(y_{1}, y_{2}\right) y_{2}^{\prime 2} .
\end{aligned}
$$


In $[18,20]$, it is shown that system (5.1) is equivalent via point transformations to the simplest equations $\ddot{u}_{1}=0, \ddot{u}_{2}=0$ if and only if

$$
S_{i}=0 \quad(i=1,2,3,4),
$$

where

$$
\begin{aligned}
& S_{1}=a_{y_{2}}-b_{y_{1}}+b e-c d, \quad S_{2}=b_{y_{2}}-c_{y_{1}}+\left(a c-b^{2}\right)+(b f-c e), \\
& S_{3}=d_{y_{2}}-e_{y_{1}}-(a e-b d)-\left(d f-e^{2}\right), \quad S_{4}=b_{y_{1}}+f_{y_{1}}-a_{y_{2}}-e_{y_{2}} .
\end{aligned}
$$

Application of fiber preserving transformation to system (5.1) also leads to the same conditions (5.2).

Example 5.2. Consider a nonlinear system

$$
y_{1}^{\prime \prime}=-y_{1}^{\prime 2}-y_{2}^{\prime 2}-q_{1}, \quad y_{2}^{\prime \prime}=q_{2}-2 y_{1}^{\prime} y_{2}^{\prime}
$$

where $q_{1}, q_{2}$ are constant. Applying the linearization criteria obtained in $[18,23]$ to system (5.4), one obtains that system (5.4) is equivalent to the free particle equations via point transformations if and only if $q_{2}=0$. Let us consider the case $q_{2} \neq 0$. Note that, for system (5.4),

$$
\lambda_{12}=-4 q_{2}, \quad \lambda_{14}=0, \quad \lambda_{15}=-4 q_{2}, \quad \lambda_{16}=0 .
$$

Since $q_{2} \neq 0$, then $\lambda_{12} \neq 0$, and (3.21) becomes $\varphi_{x x}=0$. Taking the simplest solution $\varphi=x$ of this equation and solving the compatible system of (3.5)-(3.14) for the functions $\psi_{1}$, and $\psi_{2}$, one gets the solution $\psi_{1}=(1 / 2) e^{\left(y_{1}-y_{2}\right)}$ and $\psi_{2}=(1 / 2) e^{\left(y_{1}+y_{2}\right)}$. Substituting $\varphi, \psi_{1}$ and $\psi_{2}$ into (3.18) and (3.26), one obtains $k_{1}=q_{1}+q_{2}$ and $k_{2}=q_{1}-q_{2}$. Thus, Theorem 3.2 guarantees that system (5.4) can be transformed to the system of linear equations:

$$
\ddot{u}_{1}+k_{1} u_{1}=0, \quad \ddot{u}_{2}+k_{2} u_{2}=0,
$$

and the linearizing transformation is

$$
t=x, \quad u_{1}=\frac{1}{2} e^{\left(y_{1}-y_{2}\right)}, \quad u_{2}=\frac{1}{2} e^{\left(y_{1}+y_{2}\right)}
$$

Example 5.3. A variety of applications in science and engineering such as the well-known oscillator system, the vibration of springs, and some types of conservative systems with two 
degrees of freedom are of the form

$$
y_{1}^{\prime \prime}=g_{1}(x) y_{1}+g_{2}(x) y_{2}, \quad y_{2}^{\prime \prime}=g_{3}(x) y_{2}+g_{4}(x) y_{1}
$$

For system (5.8),

$$
\lambda_{12}=-4 g_{4}, \quad \lambda_{16}=4\left(g_{1}-g_{3}\right), \quad \lambda_{15}=-4 g_{2}, \quad \lambda_{21}=-2 g_{3}, \quad \lambda_{22}=4 g_{3} \lambda_{16 x}-\lambda_{16 x x x} .
$$

Theorem 3.2 provides conditions sufficiently for system (5.8) to be reduced to a linear system with constant coefficients via a fiber preserving transformation. For example, for the oscillator system $\left(g_{2}=0, g_{4}=0\right)$, the condition is

$$
8 \lambda_{16}{ }^{3} \lambda_{21}+4 \lambda_{16}{ }^{2} \lambda_{22}+18 \lambda_{16} \lambda_{16 x x} \lambda_{16 x}-15 \lambda_{16 x}{ }^{3}=0,
$$

whereas the criteria of $[18,23]$ are only satisfied when $g_{1}=g_{3}$.

\section{Conclusion}

The necessary form of a linearizable system of two second-order ordinary differential equations $y_{1}^{\prime \prime}=f_{1}\left(x, y_{1}, y_{2}, y_{1}^{\prime}, y_{2}^{\prime}\right), y_{2}^{\prime \prime}=f_{2}\left(x, y_{1}, y_{2}, y_{1}^{\prime}, y_{2}^{\prime}\right)$ via point transformations is obtained. Some other necessary conditions were also found. Necessary and sufficient conditions for a system of two second-order ordinary differential equations to be transformed to the general form of linear system with constant coefficients via fiber preserving transformations are obtained. A linear system with constant coefficients is chosen because of its simplicity in finding the general solution. On the way of establishing of main theorems, we also give an explicit procedure for constructing this linearizing transformation.

\section{Appendix}

Consider the following:

$$
\begin{aligned}
& \lambda_{1}=2 a_{28 y_{1}}-2 a_{27 y_{2}}+a_{17} a_{25}-2 a_{18} a_{24}-a_{25} a_{28}+2 a_{26} a_{27}, \\
& \lambda_{2}=2 a_{18 y_{1}}-2 a_{17 y_{2}}-2 a_{14} a_{18}+a_{15} a_{17}-a_{15} a_{28}+2 a_{16} a_{27}, \\
& \lambda_{3}=4 a_{24 x}-2 a_{27 y_{1}}-2 a_{14} a_{27}+2 a_{17} a_{24}-2 a_{24} a_{28}+a_{25} a_{27}, \\
& \lambda_{4}=2 a_{28 y_{1}}-2 a_{25 x}+a_{15} a_{27}-2 a_{18} a_{24}, \\
& \lambda_{5}=2 a_{28 y_{2}}-4 a_{26 x}+2 a_{16} a_{27}-a_{18} a_{25}, \\
& \lambda_{6}=2 a_{25 y_{1}}-4 a_{24 y_{2}}+2 a_{14} a_{25}-2 a_{15} a_{24}+4 a_{24} a_{26}-a_{25}{ }^{2}, \\
& \lambda_{7}=4 a_{16 x}-2 a_{18 y_{2}}+a_{15} a_{18}-2 a_{16} a_{17}+2 a_{16} a_{28}-2 a_{18} a_{26},
\end{aligned}
$$




$$
\begin{aligned}
& \lambda_{8}=4 a_{16 y_{1}}-2 a_{15 y_{2}}-4 a_{14} a_{16}+a_{15}^{2}-2 a_{15} a_{26}+2 a_{16} a_{25}, \\
& \lambda_{9}=2 a_{25 y_{2}}-4 a_{26 y_{1}}-a_{15} a_{25}+4 a_{16} a_{24} \\
& \lambda_{10}=2 a_{18 x} a_{15}-8 a_{16 y_{1}} a_{19}-8 a_{16 y_{2}} a_{29}-4 a_{17 x} a_{16}-4 a_{18 x y_{2}} \\
& -4 a_{18 x} a_{26}+2 a_{18 y_{1}} a_{18}+2 a_{18 y_{2}} a_{17}+2 a_{18 y_{2}} a_{28}+8 a_{19 y_{1}} a_{16} \\
& +8 a_{19 y_{2} y_{2}}-8 a_{19 y_{2}} a_{15}+8 a_{19 y_{2}} a_{26}+4 a_{26 x} a_{18}+4 a_{28 x} a_{16} \\
& -16 a_{29} y_{2} a_{16}-2 a_{14} a_{18}{ }^{2}-2 a_{15} a_{18} a_{28}+2 a_{16} a_{17}^{2}-2 a_{16} a_{28}{ }^{2} \\
& +2 a_{17} a_{18} a_{26}+a_{18}^{2} a_{25}+2 a_{18} a_{26} a_{28} \\
& \lambda_{11}=2 a_{18 y_{2}} a_{27}-4 a_{19 y_{2}} a_{25}-4 a_{26 x x}+4 a_{26 x} a_{28}-4 a_{26 y_{1}} a_{19}-4 a_{26 y_{2}} a_{29} \\
& +4 a_{29} y_{1} a_{16}+4 a_{29} y_{2} y_{2}-4 a_{29 y_{2}} a_{26}-a_{15} a_{18} a_{27}+2 a_{16} a_{17} a_{27} \\
& -2 a_{16} a_{27} a_{28}+2 a_{18} a_{26} a_{27} \\
& \lambda_{12}=2 a_{27 x}-4 a_{29} y_{1}-a_{17} a_{27}+4 a_{19} a_{24}+2 a_{25} a_{29}-a_{27} a_{28}, \\
& \lambda_{14}=\lambda_{12}\left(a_{28}-a_{17}\right)-a_{27} \lambda_{16}-2 \lambda_{12 x} \\
& \lambda_{15}=2 a_{18 x}-4 a_{19 y_{2}}+2 a_{15} a_{19}+4 a_{16} a_{29}-a_{17} a_{18}-a_{18} a_{28}, \\
& \lambda_{16}=4 a_{19 y_{1}}-2 a_{17 x}+2 a_{28 x}-4 a_{29 y_{2}}-4 a_{14} a_{19}-2 a_{15} a_{29}+a_{17}^{2} \\
& +2 a_{19} a_{25}+4 a_{26} a_{29}-a_{28}{ }^{2}, \\
& \lambda_{17}=2 a_{19 x} a_{25}-2 a_{19 y_{2}} a_{27}+2 a_{25 x} a_{19}+4 a_{26 x} a_{29}+2 a_{28 x x}-2 a_{28 x} a_{28} \\
& -4 a_{29 x y_{2}}+4 a_{29 x} a_{26}-2 a_{29} a_{1} a_{18}+a_{15} a_{19} a_{27}+2 a_{16} a_{27} a_{29}-a_{17} a_{18} a_{27} \\
& +2 a_{18} a_{19} a_{24}+a_{18} a_{25} a_{29}-a_{18} a_{27} a_{28}-a_{27} \lambda_{15}, \\
& \lambda_{18}=2 a_{19 y_{1}} \lambda_{14}+4 a_{28 x} \lambda_{14}-10 a_{29 y_{2}} \lambda_{1} 4+2 \lambda_{14 x x}+2 \lambda_{14 x} a_{17}-2 \lambda_{14 x} a_{28} \\
& -2 a_{14} a_{19} \lambda_{14}-a_{15} a_{29} \lambda_{14}+a_{17}^{2} \lambda_{14}-a_{17} a_{28} \lambda_{14}-3 a_{18} a_{27} \lambda_{14} \\
& +5 a_{19} a_{25} \lambda_{14}+10 a_{26} a_{29} \lambda_{14}-2 a_{28}^{2} \lambda_{14} \\
& \lambda_{19}=8 a_{29} \lambda_{1} \lambda_{14} \lambda_{16}+8 \lambda_{14 x} a_{27} \lambda_{16}+18 \lambda_{14 x} \lambda_{14}+8 a_{17} a_{27} \lambda_{14} \lambda_{16}+9 a_{17} \lambda_{14}{ }^{2} \\
& -8 a_{19} a_{24} \lambda_{14} \lambda_{16}-4 a_{25} a_{29} \lambda_{14} \lambda_{16}+4 a_{27}{ }^{2} \lambda_{14} \lambda_{15}-4 a_{27} a_{28} \lambda_{14} \lambda_{16} \\
& -9 a_{28} \lambda_{14}{ }^{2} \text {, } \\
& \lambda_{20}=2 a_{28 x}-4 a_{29 y_{2}}-a_{18} a_{27}+2 a_{19} a_{25}+4 a_{26} a_{29}-a_{28}{ }^{2}, \\
& \lambda_{21}=2 a_{26 x} a_{29}+a_{28 x x}-a_{28 x} a_{28}-2 a_{29 x y_{2}}+2 a_{29 x} a_{26},
\end{aligned}
$$




$$
\begin{aligned}
& \lambda_{22}=4 a_{29} \lambda_{2} \lambda_{16 x}-2 a_{28 x} \lambda_{16 x}-\lambda_{16 x x x}-4 \lambda_{16 x} a_{26} a_{29}+\lambda_{16 x} a_{28}{ }^{2}, \\
& \lambda_{23}=2 a_{28 x}-4 a_{29} y_{2}+4 a_{26} a_{29}-a_{28}{ }^{2}, \\
& \lambda_{24}=32\left(a_{19 x y_{1}}-a_{19 x} a_{14}\right)-16\left(a_{17 y_{1}} a_{19}+a_{18 y_{1}} a_{29}+a_{29 x} a_{15}-a_{14} a_{18} a_{29}\right) \\
& +56\left(a_{19 y_{1}} a_{17}-a_{14} a_{17} a_{19}\right)-24\left(a_{19 y_{1}} a_{28}+a_{14} a_{19} a_{28}\right)+21 a_{28}^{3} \\
& +160\left(a_{26 x} a_{29}-a_{29 x y_{2}}+a_{29 x} a_{26}\right)+120\left(a_{17} a_{26} a_{29}-a_{29 y_{2}} a_{17}\right) \\
& +64 a_{28 x x}+48 a_{28 x} a_{17}-112 a_{28 x} a_{28}+88\left(a_{29} y_{2} a_{28}-a_{26} a_{28} a_{29}\right) \\
& -36 a_{15} a_{17} a_{29}+20 a_{15} a_{28} a_{29}+15 a_{17}^{3}-9 a_{17}^{2} a_{28}-27 a_{17} a_{28}^{2} \text {, } \\
& \lambda_{25}=12\left(\lambda_{15 x x} a_{28}-\lambda_{15 x x} a_{17}+\lambda_{15 x} a_{15} a_{29}\right)+24\left(\lambda_{15 x} a_{14} a_{19}-a_{19} \lambda_{1} \lambda_{15 x}\right) \\
& -16 \lambda_{15 x x x}-32 a_{28 x} \lambda_{15 x}-9 \lambda_{15 x} a_{17}{ }^{2}+6 \lambda_{15 x} a_{17} a_{28}+19 \lambda_{15 x} a_{28}{ }^{2} \\
& +88\left(a_{29 y_{2}} \lambda_{15 x}-\lambda_{15 x} a_{26} a_{29}\right), \\
& \lambda_{26}=\left(\lambda _ { 1 5 } ^ { 2 } \left(16 a_{19 y_{1}}+32 a_{28 x}-80 a_{29} y_{2}-16 a_{14} a_{19}\right.\right. \\
& \left.-8 a_{15} a_{29}+5 a_{17}^{2}-2 a_{17} a_{28}+80 a_{26} a_{29}-19 a_{28}^{2}\right) \\
& \left.+4 \lambda_{15}\left(\lambda_{15 x} a_{28}-4 \lambda_{15 x x}-\lambda_{15 x} a_{17}\right)+20 \lambda_{15 x}{ }^{2}\right) /\left(32 \lambda_{15}^{2}\right), \\
& \lambda_{27}=8 a_{14 y_{1}} a_{19}+4 a_{15 y_{1}} a_{29}+4 a_{17 x y_{1}}-4 a_{17 y_{1}} a_{17}+4 a_{18 x} a_{24}-2 a_{18 y_{1}} a_{27} \\
& -8 a_{19} y_{1} y_{1}+8 a_{19} y_{1} a_{14}-8 a_{19 y_{2}} a_{24}-4 a_{24 x} a_{18}-2 a_{27 x} a_{15}+8 a_{29} y_{1} a_{15} \\
& +2 a_{14} a_{18} a_{27}+a_{15} a_{17} a_{27}-2 a_{15} a_{25} a_{29}+a_{15} a_{27} a_{28}+8 a_{16} a_{24} a_{29} \\
& -4 a_{17} a_{18} a_{24}-a_{18} a_{25} a_{27} \text {, } \\
& \lambda_{28}=2 a_{17 y_{1}} a_{27}-8 a_{19 y_{1}} a_{24}-4 a_{24 x x}+4 a_{24 x} a_{28}-4 a_{24 y_{1}} a_{19} \\
& -4 a_{24 y_{2}} a_{29}+4 a_{29} y_{1} y_{1}+4 a_{29} y_{1} a_{14}-4 a_{29 y_{1}} a_{25}+4 a_{29} y_{2} a_{24} \\
& -a_{15} a_{27}^{2}+2 a_{18} a_{24} a_{27} \\
& \lambda_{29}=2 a_{18 y_{1}} a_{27}-2 a_{19 y_{1}} a_{25}-4 a_{19 y_{2}} a_{24}-4 a_{24 y_{2}} a_{19}-2 a_{25 x x}+2 a_{25 x} a_{28} \\
& -4 a_{26 y_{1}} a_{29}+4 a_{29} y_{1} y_{2}+2 a_{29} y_{1} a_{15}-4 a_{29} y_{1} a_{26}-2 a_{14} a_{18} a_{27} \\
& +2 a_{14} a_{19} a_{25}+a_{15} a_{17} a_{27}-2 a_{15} a_{19} a_{24}-a_{15} a_{25} a_{29}-a_{15} a_{27} a_{28} \\
& +4 a_{16} a_{24} a_{29}+a_{18} a_{25} a_{27}+4 a_{19} a_{24} a_{26}-a_{19} a_{25}{ }^{2}, \\
& \mu_{1}=2 a_{28 x}-4 a_{29} y_{2}+4 a_{26} a_{29}-a_{28}{ }^{2} \text {, }
\end{aligned}
$$


ISRN Mathematical Analysis

$$
\begin{aligned}
& \mu_{2}=4 a_{19} y_{1}-2 a_{17 x}-4 a_{14} a_{19}+a_{17^{2}}, \\
& a_{29} y_{1} \mu_{3}=\frac{2 a_{29 x y_{1}}+a_{29 y_{1}} a_{17}-a_{29 y_{1}} a_{28}}{4}, \\
& a_{19 y_{2}} \mu_{4}=\frac{2 a_{19 x y_{2}}-a_{19 y_{2}} a_{17}+a_{19 y_{2}} a_{28}}{4}, \\
& \mu_{5}=-\left(\mu_{1}+\mu_{2}\right) \\
& \omega_{1}=2 a_{12 y_{1}}-4 a_{11 y_{2}}-2 a_{11} a_{15}+2 a_{12} a_{14}-a_{12} a_{25}+4 a_{13} a_{24} \\
& \omega_{2}=2 a_{12 y_{2}}-4 a_{13 y_{1}}+4 a_{11} a_{16}-a_{12} a_{15}+2 a_{12} a_{26}-2 a_{13} a_{25} \\
& \omega_{3}=a_{15 y_{1}}-2 a_{14 y_{2}}-a_{25 y_{2}}+2 a_{26 y_{1}}-4 a_{11} a_{18}+2 a_{12} a_{17} \\
& -2 a_{12} a_{28}+4 a_{13} a_{27}, \\
& \omega_{4}=2 a_{15 y_{2}}-4 a_{13 x}-4 a_{16 y_{1}}-2 a_{13} a_{17}-2 a_{13} a_{28}+4 a_{14} a_{16} \\
& -a_{15}^{2}+2 a_{15} a_{26}-2 a_{16} a_{25} \\
& \omega_{5}=4 a_{12 x}+4 a_{25 y_{2}}-8 a_{26 y_{1}}+8 a_{11} a_{18}-2 a_{12} a_{17}+6 a_{12} a_{28}-8 a_{13} a_{27} \\
& -2 a_{15} a_{25}+8 a_{16} a_{24}+\omega_{3} \\
& \omega_{6}=4 a_{11 x}+4 a_{24 y_{2}}-2 a_{25 y_{1}}+2 a_{11} a_{17}+2 a_{11} a_{28}-2 a_{14} a_{25}+2 a_{15} a_{24} \\
& -4 a_{24} a_{26}+a_{25}{ }^{2} \\
& \omega_{7}=2 a_{18 y_{2}}-4 a_{16 x}-4 a_{13} a_{19}-a_{15} a_{18}+2 a_{16} a_{17}-2 a_{16} a_{28}+2 a_{18} a_{26}, \\
& \omega_{8}=5 a_{15 x}-a_{17 y_{2}}-4 a_{18 y_{1}}-6 a_{26 x}+3 a_{28 y_{2}}+4 a_{12} a_{19}-8 a_{13} a_{29} \\
& +4 a_{14} a_{18}-2 a_{15} a_{17}+2 a_{15} a_{28}+4 a_{16} a_{27}-4 a_{18} a_{25} \\
& \omega_{9}=a_{17 y_{1}}-2 a_{14 x}+3 a_{25 x}-3 a_{28 y_{1}}+4 a_{12} a_{29}-2 a_{15} a_{27}+4 a_{18} a_{24}, \\
& \omega_{10}=2 a_{27} y_{1}-4 a_{24 x}-4 a_{11} a_{29}+2 a_{14} \mathrm{a}_{27}-2 a_{17} a_{24}+2 a_{24} a_{28}-a_{25} a_{27}, \\
& \omega_{11}=4 a_{25 x}+4 a_{27 y_{2}}-8 a_{28 y_{1}}+8 a_{11} a_{19}+8 a_{12} a_{29}-2 a_{15} a_{27}-2 a_{17} a_{25} \\
& +8 a_{18} a_{24}+2 a_{25} a_{28}-4 a_{26} a_{27} \\
& \omega_{12}=12 a_{17 y_{2}}-12 a_{18 y_{1}}-8 a_{26 x}+4 a_{28 y_{2}}-8 a_{12} a_{19}-24 a_{13} a_{29}+12 a_{14} a_{18} \\
& -6 a_{15} a_{17}+6 a_{15} a_{28}-8 a_{16} a_{27}-2 a_{18} a_{25} \\
& \omega_{13}=2 \lambda_{15}, \quad \omega_{14}=4 \lambda_{16}, \quad \omega_{15}=-2 \lambda_{12} .
\end{aligned}
$$




\section{Acknowledgments}

This research was supported by the Royal Golden Jubilee Ph.D. Program of Thailand (TRF). The authors are also thankful to E. Schulz for fruitful discussions.

\section{References}

[1] S. Lie, "Klassifikation und integration von gewohnlichen differential gleichungen zwischen $x, y$, die eine gruppe von transformationen gestatten. III," Archiv for Mathematik og Naturvidenskab, vol. 8, no. 4, pp. 371-427, (1883), reprinted in Lie's Gessammelte Abhandlungen 5, paper XIY, pp. 362-427, 1924.

[2] R. Liouville, "Sur les invariants de certaines equations differentielles et sur leurs applications," Journal de l'Ecole Polytechnique, vol. 59, pp. 7-76, 1889.

[3] A. M. Tresse, Détermination des Invariants Ponctuels de L'équation Différentielle Ordinaire du Second Ordre $y^{\prime \prime}=\omega\left(x, y, y^{\prime}\right)$, Preisschriften der Fürstlichen Jablonowski'schen Gesellschaft XXXII, Leipzig, S.Herzel, 1896.

[4] E. Cartan, "Sur les variétés à connexion projective," Bulletin de la Société Mathématique de France, vol. 52, pp. 205-241, 1924.

[5] S.-S. Chern, "The geometry of the differential equation $y^{\prime \prime \prime}=f\left(x, y, y^{\prime}, y^{\prime \prime}\right)$," National Tsing Hua University, vol. 4, pp. 97-111, 1940.

[6] G. Grebot, "The characterization of third order ordinary differential equations admitting a transitive fiber-preserving point symmetry group," Journal of Mathematical Analysis and Applications, vol. 206, no. 2, pp. 364-388, 1997.

[7] S. Neut and M. Petitot, "La géométrie de l'équation $y^{\prime \prime \prime}=f\left(x, y, y^{\prime}, y^{\prime \prime}\right)$," Comptes Rendus de L'Académie des Sciences Paris, vol. 335, no. 6, pp. 515-518, 2002.

[8] N. Euler, T. Wolf, P. G. L. Leach, and M. Euler, "Linearisable third-order ordinary differential equations and generalised Sundman transformations: the case $X^{\prime \prime \prime}=0, "$ Acta Applicandae Mathematicae, vol. 76, no. 1, pp. 89-115, 2003.

[9] N. Euler and M. Euler, "Sundman symmetries of nonlinear second-order and third-order ordinary differential equations," Journal of Nonlinear Mathematical Physics, vol. 11, no. 3, pp. 399-421, 2004.

[10] N. H. Ibragimov and S. V. Meleshko, "Linearization of third-order ordinary differential equations," Archives of ALGA, vol. 1, pp. 71-92, 2004.

[11] N. H. Ibragimov and S. V. Meleshko, "Linearization of third-order ordinary differential equations by point and contact transformations," Journal of Mathematical Analysis and Applications, vol. 308, no. 1, pp. 266-289, 2005.

[12] S. V. Meleshko, "On linearization of third-order ordinary differential equations," Journal of Physics $A$, vol. 39, no. 49, pp. 15135-15145, 2006.

[13] N. H. Ibragimov, S. V. Meleshko, and S. Suksern, "Linearization of fourth-order ordinary differential equations by point transformations," Acta Applicandae Mathematicae, vol. 23, no. 41, Article ID 235206, 2008.

[14] S. Suksern, N. H. Ibragimov, and S. V. Meleshko, "Criteria for fourth-order ordinary differential equations to be linearizable by contact transformations," Communications in Nonlinear Science and Numerical Simulation, vol. 14, no. 6, pp. 2619-2628, 2009.

[15] W. Nakpim and S. V. Meleshko, "Linearization of third-order ordinary differential equations by generalized Sundman transformations: the case $X^{\prime \prime \prime}+\alpha X=0,{ }^{\prime \prime}$ Communications in Nonlinear Science and Numerical Simulation, vol. 15, no. 7, pp. 1717-1723, 2010.

[16] W. Nakpim and S. V. Meleshko, "Linearization of second-order ordinary differential equations by generalized Sundman transformations," Symmetry, Integrability and Geometry, vol. 6, p. 51, 2010.

[17] S. V. Meleshko, Methods for Constructing Exact Solutions of Partial Differential Equations, Springer, New York, NY, USA, 2005.

[18] A. V. Aminova and N. A.-M. Aminov, "Projective geometry of systems of second-order differential equations," Sbornik, vol. 197, no. 7, pp. 951-955, 2006.

[19] Soh C. Wafo and F. M. Mahomed, "Linearization criteria for a system of second-order ordinary differential equations," International Journal of Non-Linear Mechanics, vol. 36, no. 4, pp. 671-677, 2001.

[20] F. M. Mahomed and A. Qadir, "Linearization criteria for a system of second-order quadratically semilinear ordinary differential equations," Nonlinear Dynamics, vol. 48, no. 4, pp. 417-422, 2007. 
[21] S. Sookmee, Invariants of the equivalence group of a system of second-order ordinary differential equations, M.Sc. thesis, Nakhon Ratchasima, Thailand, 2005.

[22] S. Sookmee and S. V. Meleshko, "Conditions for linearization of a projectable system of two secondorder ordinary differential equations," Journal of Physics A, vol. 41, no. 40, Article ID 402001, 7 pages, 2008.

[23] S. Neut, M. Petitot, and R. Dridi, "Élie Cartan's geometrical vision or how to avoid expression swell," Journal of Symbolic Computation, vol. 44, no. 3, pp. 261-270, 2009.

[24] Y. Yu. Bagderina, "Linearization criteria for a system of two second-order ordinary differential equations," Journal of Physics A, vol. 43, no. 46, Article ID 465201, 2010. 


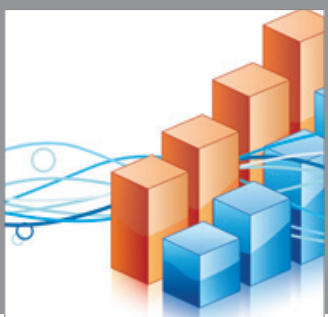

Advances in

Operations Research

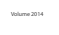

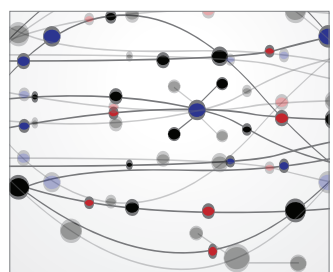

\section{The Scientific} World Journal
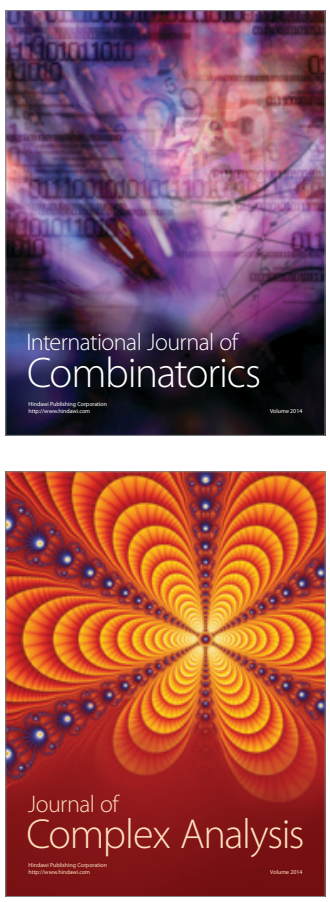

International Journal of

Mathematics and

Mathematical

Sciences
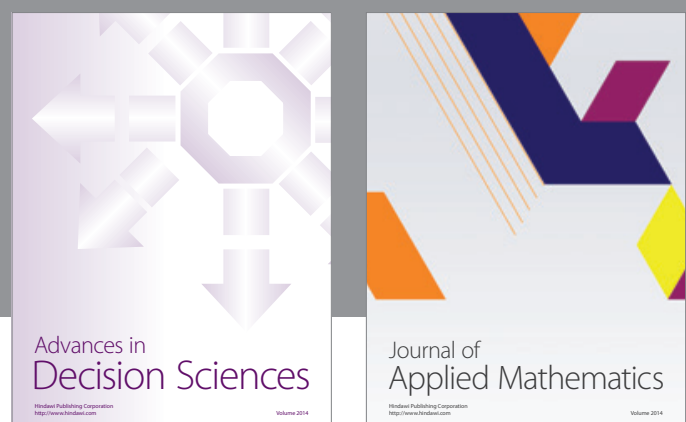

Journal of

Applied Mathematics
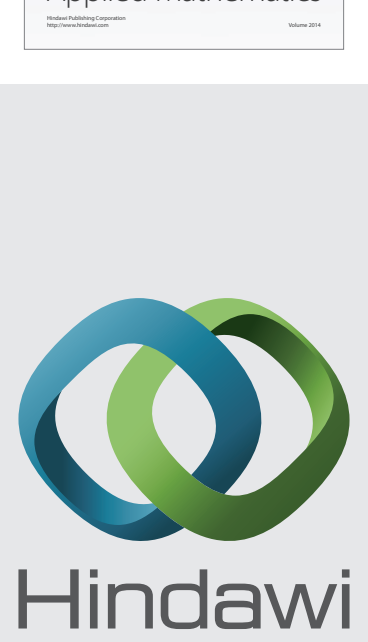

Submit your manuscripts at http://www.hindawi.com
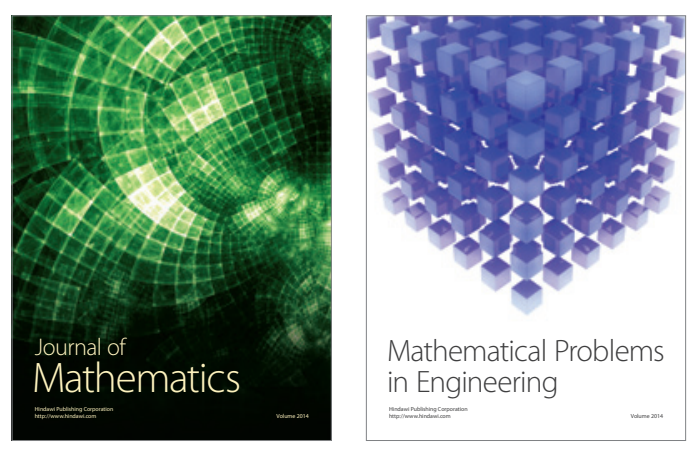

Mathematical Problems in Engineering
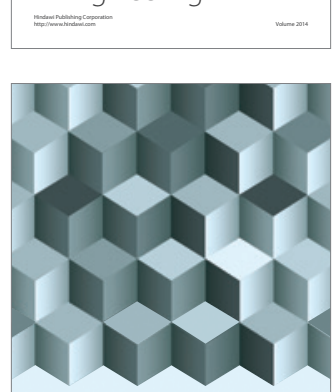

Journal of

Function Spaces
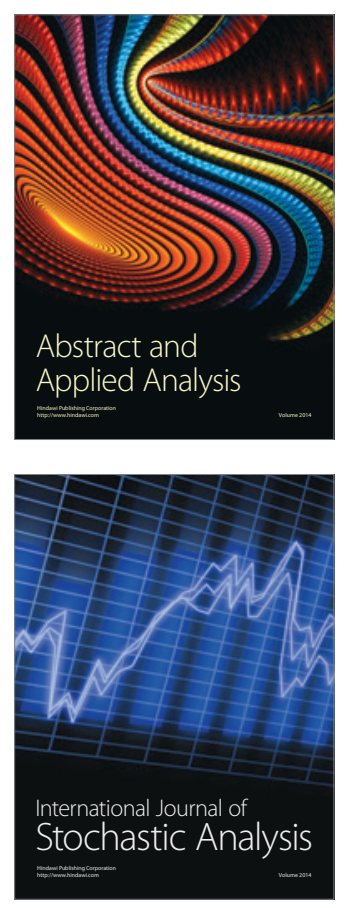

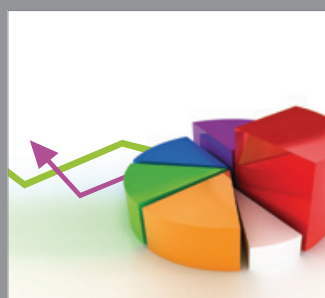

ournal of

Probability and Statistics

Promensencen
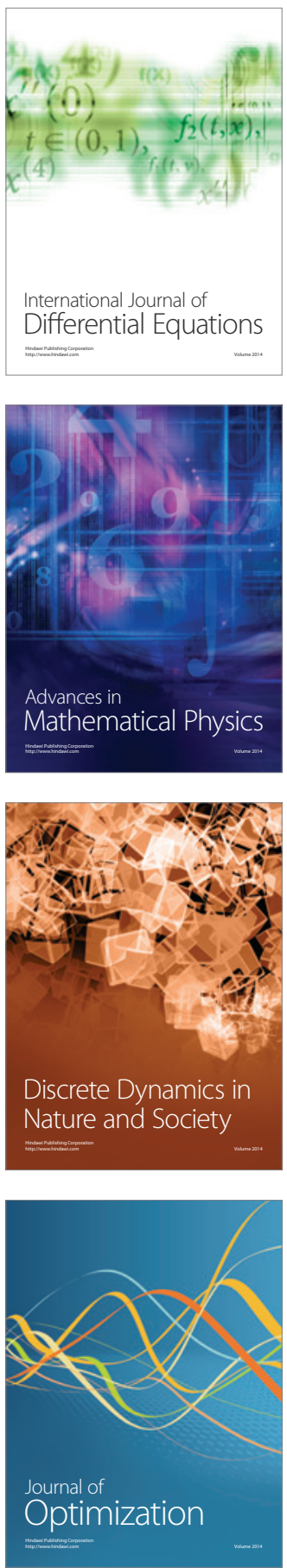